\title{
Assessment of serum asymmetric dimethyl arginine levels in patients with coronary artery ectasia
}

\author{
Oğuzhan Çelik ${ }^{1}$, Burhan Çabuk², Erkan Demirci ${ }^{3}$, Macit Kalçık $^{4 *}$, Lütfü Bekar ${ }^{4}$, Mucahit Yetim $^{4}$ and Tolga Doğan $^{4}$ \\ ${ }^{1}$ Department of Cardiology, Muğla Sıtkı Koçman University Training and Research Hospital, Muğla, Turkey \\ ${ }^{2}$ Department of Cardiology, Medinova Hospital, Aydın, Turkey \\ ${ }^{3}$ Department of Cardiology, Kayseri Training and Research Hospital, Kayseri, Turkey \\ ${ }^{4}$ Department of Cardiology, Hitit University Faculty of Medicine, Çorum, Turkey
}

\begin{abstract}
Background: Previous studies have demonstrated that asymmetric dimethyl arginine (ADMA) levels were strongly associated with cardiovascular diseases including endothelial dysfunction and atherosclerosis. Coronary artery ectasia (CAE) has been characterized as a localized or diffuse non-obstructive lesion of the epicardial coronary arteries with a luminal dilation exceeding 1.5 -fold the normal adjacent segment or vessel diameter. The etiopathogenesis of this coronary enlargement is completely unknown, and its clinical significance also remains poorly understood. In this study, we aimed to investigate the role of serum ADMA levels in the pathogenesis of CAE.
\end{abstract}

Methods: Twenty-seven patients with CAE (female: $51.9 \%$; mean age: $60.2 \pm 7.4$ years) and 29 controls (female: $55.2 \%$; mean age: $59.3 \pm 6.9$ years) with normal coronary flow were included in this study. CAE was classified according to its extent and number of vessels involved (Markis classification). Serum ADMA levels were determined by using enzymatic assays from venous blood samples.

Results: There was no significant difference in terms of demographic parameters between the patients with CAE and the controls. Serum ADMA levels were higher in the CAE group, however this was not statistically significant (1.22 \pm 0.13 vs. $1.16 \pm 0.12 \mu \mathrm{M} / \mathrm{L}$; $\mathrm{p}=0.091)$. In subgroup analysis, serum ADMA levels were significantly higher in patients with diffuse $\mathrm{CAE}$ as compared to controls $(1.25 \pm 0.11$ vs. $1.16 \pm 0.12 \mu \mathrm{M} / \mathrm{L} ; \mathrm{p}=0.024)$.

Conclusion: Increased ADMA levels may play an important role in the pathogenesis of diffuse CAE. Further large-scale studies are required to determine the relationship between ADMA levels and CAE. These findings suggest that increased ADMA level may be associated with endothelial dysfunction leading to the development of CAE.

\section{Introduction}

Coronary artery ectasia (CAE) is defined as dilatation of the coronary artery 1.5-times greater than that of an adjacent normal segment [1]. Angiographic frequency of CAE has been reported to be $0.3 \%-5.3 \%$ in various series [2]. The etiopathogenesis of this clinical entity is not fully understood. Previous studies have found that atherosclerosis and inflammation play a key role in the development of CAE, although the reasons for the formation of ectasia have not been understood exactly [3]. Kawasaki disease, congenital anomalies, collagen tissue diseases, and connective tissue diseases are other common causes of CAE [4]. Chest pain is seen as the primary symptom of CAE. However, dysrhythmias, acute coronary syndromes, and sudden cardiac death are other clinical conditions that are observed in CAE [5]. In addition, several studies have demonstrated a relationship between CAE and non-coronary aneurysms, including aortic, popliteal, and pulmonary aneurysms [6].

Nitric oxide (NO) is well-known to be a vasodilator that relaxes vascular smooth muscle via the cyclic guanosine monophosphate dependent pathway. NO is produced from its precursor, L-arginine, by endothelial NO synthase enzymes. Asymmetric dimethyl arginine (ADMA) is an endogenous inhibitor of all major isoforms of NO synthase, which reduces NO release from the endothelium, and has been associated with endothelial dysfunction and atherosclerosis [7]. Increased ADMA level is also an independent risk factor for progression of atherosclerosis and total mortality in coronary artery disease [8]. Recently, elevated serum levels of ADMA have been demonstrated to be associated with cardiovascular disease and many of the risk factors related with endothelial dysfunction such as hypercholesterolemia, hypertension, smoking and diabetes [9].

In this study, we have tested the hypothesis that endothelial dysfunction may play a role in the pathogenesis of CAE. Therefore, we aimed to investigate serum ADMA levels in patients with and without CAE.

${ }^{*}$ Correspondence to: Macit Kalcik, Department of Cardiology, Hitit University Faculty of Medicine, Çorum, Turkey, Tel: (90)536 4921789, E-mail: macitkalcik@yahoo.com

Key words: asymmetric dimethyl arginine, coronary artery ectasia, coronary angiography

Received: March 01, 2019; Accepted: March 06, 2019; Published: March 15, 2019 


\section{Methods}

\section{Study population}

This single-center study enrolled 27 angiographically identified patients with CAE (female: $51.9 \%$; mean age: $60.2 \pm 7.4$ years), along with 29 age and sex matched control participants with angiographically proven normal coronary arteries without CAE (female: $55.2 \%$; mean age: $59.3 \pm 6.9$ years). All study participants referred to coronary angiography due to the presence of angina, angina-like chest pain, or positive results of stress test for myocardial ischemia. Patients with a history of myocardial infarction, coronary artery bypass graft surgery or percutaneous coronary intervention, left ventricular dysfunction (left ventricular ejection fraction $<50 \%$ ), severe heart valve disease, cardiomyopathy, arrhythmia, left ventricular hypertrophy, connective tissue disease and liver, kidney or thyroid dysfunction were excluded from the study. All patients were underwent transthoracic echocardiography (TTE). Complete blood count and blood chemistry panel were carried out in all patients at the time of admission. All demographic, laboratory and echocardiographic parameters were recorded into a dataset and compared between CAE patients and controls. All patients provided a written informed consent and the study protocol was approved by the local ethics committee of the hospital in accordance with the Declaration of Helsinki and Good Clinical Practice guidelines.

\section{Diagnosis and classification of CAE}

Coronary angiography was done by clinical indications such as abnormal stress test results, positive treadmill test, dobutamine stress echo, typical chest pain, or signs of ischemia during myocardial perfusion scintigraphy. All study population underwent selective coronary artery angiography after appropriate patient preparation. Femoral artery and sometimes radial or brachial artery cannulation was used for the arterial access site and a Judkins system was applied for cannulation of the left and right coronary arteries. Coronary angiographies were evaluated by at least two independent interventional cardiologists. Coronary artery diameters were measured by quantitative angiography on digital angiograms. Angiograms with localised and/ or diffuse dilatation without a stenotic lesion exceeding 1.5 times the normal segment of the artery were diagnosed as CAE (Figure 1). When an identifiable normal adjacent segment could not be found, the mean diameter of the corresponding coronary segment in the control group was used as the normal value. CAE without coronary artery stenosis was considered as isolated CAE, and the severity of isolated CAE was determined according to the Markis classification [10]. In decreasing order of severity, diffuse ectasia of two or three vessels was classified as type 1, diffuse disease in one vessel and localised disease in another

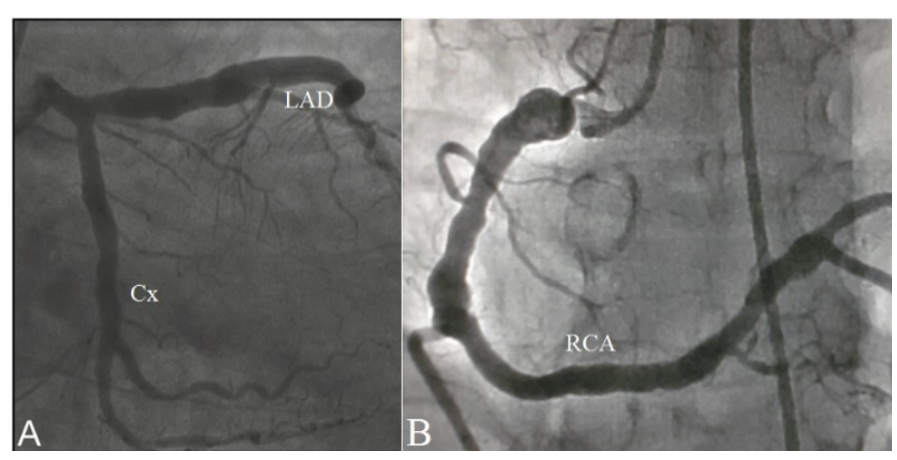

Figure 1. An example coronary angiography revealing diffuse coronary artery ectasia (Markis type I) in all coronary arteries (LAD: Left anterior descending coronary artery, Cx: Left circumflex coronary artery RCA: Right coronary artery) vessel as type 2, diffuse ectasia of only one vessel as type 3 , and localised segmental ectasia as type 4 .

\section{Laboratory analysis}

In order to detect serum ADMA levels, venous blood samples were collected after 12-hours of fasting by a clean puncture of an antecubital vein from all patients. Blood samples were taken into ethylenediaminetetraaceticacid (EDTA) containing tubes and centrifuged at $3000 \mathrm{rpm}$ for 10 minutes at room temperature. The plasma samples, obtained after centrifugation, were stored at $-20^{\circ} \mathrm{C}$, for further analysis. Serum ADMA levels were measured by enzyme linked immunosorbent assay (ELISA) method by using an Immunodiagnostic human ADMA kit (Immunodiagnostic AG, Bensheim, Germany). Complete blood counting's were measured on Sysmex XT2000i analyser (Sysmex corporation, Kobe, Japan). Fasting blood glucose, urea, creatinine, aspartate transaminase (AST), alanine transaminase (ALT), sodium, potassium, total cholesterol (TC), high-density lipoprotein (HDL), low-density lipoprotein (LDL), and triglyceride (TG) levels were also measured on an autoanalyzer (Siemens Advia 2400 Chemistry System, Siemens Diagnostic, Tarrytown, USA).

\section{Statistical analysis}

Statistical analyses were performed using IBM SPSS Statistics for Windows, Version 19.0. (IBM Corp. Armonk, NY). The variables were investigated using analytical methods (KolmogorovSmirnov/Shapiro-Wilk test) to determine whether or not they were approximately normally distributed. Descriptive statistics were reported as mean with standard deviation for continuous variables with normal distribution, median and $25^{\text {th }}-75^{\text {th }}$ percentile values for continuous variables without normal distribution, and frequencies with percentages for the categorical variables. Group comparisons for continuous variables were tested using Student $t$ test when data distribution was normal and using Mann-Whitney $U$ test when data distributions were not normal. Comparisons for categorical variables were evaluated by Chi-square test. Significance level was accepted as $\mathrm{p}<0.05$ in all statistical analyses.

\section{Results}

The clinical and demographical characteristics of patients with and without CAE were presented in (Table 1). There was no significant difference between the groups in terms of age, gender, body mass index, frequency of diabetes mellitus, hypertension, dyslipidemia, smoking status and family history of coronary artery disease. Heart rate, systolic blood pressure, diastolic blood pressure and left ventricular ejection fraction were also similar between the groups. Upon comparison of laboratory parameters between CAE group and controls, there was no significant difference in terms of white blood cells, platelets, hemoglobin, fasting blood glucose, urea, creatinine, AST, ALT, sodium, potassium, TC, HDL, LDL, and TG levels between the groups (Table 1).

Patients with CAE were classified according to the Markis classification and there were $5(19 \%)$ patients with type I CAE, 8 (30\%) patients with type II CAE, 2 (7\%) patients with type III CAE, and 12 (44\%) patients with type IV CAE (Figure 2A). The most common type of CAE was type IV among patients with CAE. Involvement of coronary arteries in patients with CAE were also evaluated. Left main coronary artery was involved in $3(11 \%)$ patients, left anterior descending coronary artery was involved in 10 (37\%) patients, left circumflex coronary artery was involved in 12 (44\%) patients, and right coronary artery was involved in 14 (52\%) patients. The most commonly 
involved coronary artery among patients with CAE was right coronary artery (Figure 2B).

Serum ADMA levels were higher in the CAE group, however this was not statistically significant $(1.22 \pm 0.13 v$ s. $1.16 \pm 0.12 \mu \mathrm{M} / \mathrm{L} ; \mathrm{p}=0.091)$ (Figure 3A). Then, patients with CAE were reclassified into 2 subgroups as patients with (type I, II and III) and without (Type IV) diffuse CAE. In subgroup analysis, serum ADMA levels were significantly higher in patients with diffuse CAE as compared to controls (1.25 \pm 0.11 vs. 1.16 $\pm 0.12 \mu \mathrm{M} / \mathrm{L} ; \mathrm{p}=0.024$ ) (Figure 3B).

\section{Discussion}

In this study we have focused on the clinical features and angiographic characteristics and the role of serum ADMA levels in patients with CAE. The results demonstrated that increased ADMA

Table 1. Comparison of demographic and laboratory parameters between patient groups with and without coronary artery ectasia

\begin{tabular}{|c|c|c|c|}
\hline & CAE $(n=27)$ & $\begin{array}{c}\text { Controls } \\
(\mathrm{n}=29)\end{array}$ & $P$ value \\
\hline Age (years) & $60.2 \pm 7.4$ & $59.3 \pm 6.9$ & 0.635 \\
\hline Gender (female), n(\%) & $14(51.9)$ & $16(55.2)$ & 0.984 \\
\hline Body mass index $\left(\mathrm{kg} / \mathrm{m}^{2}\right)$ & $28.6 \pm 5.07$ & $29.1 \pm 2.93$ & 0.740 \\
\hline Hypertension, $\mathrm{n}(\%)$ & $8(29.6)$ & $8(27.6)$ & 0.866 \\
\hline Diabetes Mellitus, n(\%) & $4(14.8)$ & $3(10.3)$ & 0.700 \\
\hline Dyslipidemia, $\mathrm{n}(\%)$ & $6(22.2)$ & $5(17.2)$ & 0.895 \\
\hline Smoking, $\mathrm{n}(\%)$ & $6(22.2)$ & $8(27.6)$ & 0.877 \\
\hline Family history of CAD, n(\%) & $10(37)$ & $7(24.1)$ & 0.448 \\
\hline Systolic blood pressure (mmHg) & $129.1 \pm 10.9$ & $127.6 \pm 12.1$ & 0.632 \\
\hline Diastolic blood pressure (mmHg) & $76.6 \pm 7.7$ & $80.2 \pm 8.1$ & 0.095 \\
\hline Heart Rate (bpm) & $71.1 \pm 8.7$ & $74 \pm 8.2$ & 0.203 \\
\hline LV EF $(\%)$ & $61.7 \pm 3.6$ & $62.1 \pm 3.1$ & 0.673 \\
\hline White blood cell $\left(\times 10^{3} / \mathrm{mL}\right)$ & $7.29 \pm 1.81$ & $7.50 \pm 2.49$ & 0.737 \\
\hline Platelets $\left(\mathrm{x} 10^{3} / \mathrm{mL}\right)$ & $233.84 \pm 59.06$ & $230.96 \pm 58.21$ & 0.863 \\
\hline Hemoglobin (mg/dL) & $11.28 \pm 2.01$ & $11.99 \pm 1.81$ & 0.193 \\
\hline Glucose (mg/dL) & $101.8 \pm 8.7$ & $99.1 \pm 10.3$ & 0.308 \\
\hline Urea $(\mathrm{mg} / \mathrm{dL})$ & $15.3 \pm 3.6$ & $14.6 \pm 3.9$ & 0.523 \\
\hline Creatinine $(\mathrm{mg} / \mathrm{dL})$ & $1.0 \pm 0.2$ & $0.9 \pm 0.2$ & 0.332 \\
\hline Aspartate transaminase $(\mathrm{u} / \mathrm{L})$ & $29(22-42)$ & $31(19.5-63)$ & 0.393 \\
\hline Alanine transaminase $(\mathrm{u} / \mathrm{L})$ & $17(14-30.5)$ & $17(11.5-41)$ & 0.719 \\
\hline Sodium $(\mathrm{mEq} / \mathrm{L})$ & $137(135-139)$ & $137(135-140)$ & 0.953 \\
\hline Potasium (mEq/L) & $4.42 \pm 0.57$ & $4.24 \pm 0.54$ & 0.242 \\
\hline Total cholesterol (mg/dL) & $112 \pm 28$ & $115 \pm 23$ & 0.671 \\
\hline High density lipoprotein (mg/dL) & $43.1 \pm 8.0$ & $45.5 \pm 10.3$ & 0.338 \\
\hline Low density lipoprotein (mg/dL) & $165 \pm 53$ & $153 \pm 48$ & 0.376 \\
\hline Triglyceride $(\mathrm{mg} / \mathrm{dL})$ & $186 \pm 39$ & $189 \pm 27$ & 0.791 \\
\hline $\begin{array}{l}\text { Asymmetric dimethyl arginine } \\
(\mu \mathrm{M} / \mathrm{L})\end{array}$ & $1.22 \pm 0.13$ & $1.16 \pm 0.12$ & 0.091 \\
\hline
\end{tabular}

CAD: Coronary artery disease, CAE: Coronary artery ectasia, LVEF: Left ventricular ejection fraction

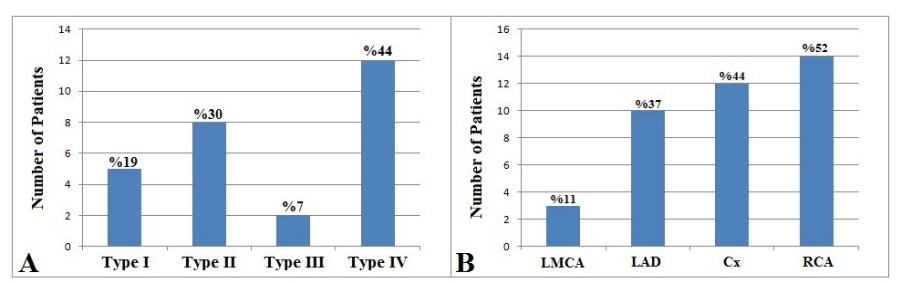

Figure 2. Angiographic characteristics of patients in the coronary ectasia group. Distribution of the patients according to Markis classification (A) and involvement of coronary arteries in patients with coronary artery ectasia (B). (LMCA: Left main coronary artery, LAD: Left anterior descending coronary artery, Cx: Left circumflex coronary artery RCA: Right coronary artery)

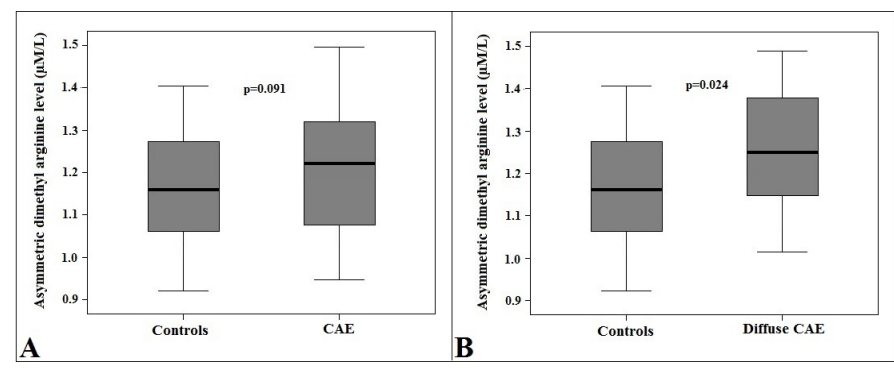

Figure 3. Comparison of box-plot graphs of serum asymmetric dimethyl arginine levels between patients with and without coronary artery ectasia (A) and between patients with diffuse coronary artery ectasia and controls (B). (CAE: Coronary artery ectasia)

levels may play an important role in the pathogenesis of diffuse CAE. These findings suggest that increased ADMA level may be associated with endothelial dysfunction leading to the development of CAE.

CAE is not a very rare finding during coronary angiography, which is characterized by abnormal coronary dilatation. Coronary blood flow in ectatic segments is impaired even in the absence of obstructive coronary artery disease. The mechanism of myocardial ischemia in CAE without coexisting coronary artery stenosis is unknown. There is evidence suggesting that endothelial dysfunction contributes to microvascular dysfunction [11]. Although the precise mechanism of endothelial dysfunction is not clear in CAE; changes in flow properties in the dilated segments with subsequent alterations in blood viscosity, activation of the coagulation system and distal coronary embolization of material originated in the ectatic segment may explain the possible underlying ischemia [12].

Endothelial dysfunction is one of the most significant early indicators of atherosclerotic processes [13]. The normal function of the endothelium layer is dependent on the balance between the endothelium-derived relaxing factors and the endothelium-derived constrictor factors. The most important of the endothelium-derived mediators is NO. A decrease in NO production or activity, accompanied by an increase in the synthesis of oxygen species free radicals is the main mechanism for endothelial dysfunction and increases the risk for the development of atherosclerosis [14].

Endothelial dysfunction is one of the earliest events in the pathogenesis of cardiovascular diseases before the documentation of atherosclerotic plaques by angiography. Over the last decade, evidence has accumulated from clinical and experimental studies for a close association of elevated serum concentrations of ADMA and vascular endothelial dysfunction. ADMA was considered as an indicator of early atherosclerosis which reduces vascular compliance, increases vascular resistance, and limits blood flow by inhibiting NO synthesis [15]. The major findings of our study were that the patients with diffuse CAE have higher serum concentrations of ADMA levels as compared to controls. These results further strengthen the concept that vascular endothelial function is impaired in patients with CAE.

Several studies have demonstrated that the right coronary artery was the most commonly involved vessel in the setting of CAE [16-18], whereas others have reported that the left anterior descending coronary artery was the vessel most commonly affected $[19,20]$. In our study, the right coronary artery was the most commonly involved vessel.

Although this study was not designed to investigate the mechanism by which ADMA contributes to CAE, it may be concluded that, by impairing coronary blood flow, elevated serum concentrations of ADMA may be responsible for the myocardial 
ischemic symptoms and the positive results of stress test for myocardial ischemia in patients with CAE. Thus, it may be suggested that ADMA reducing therapies such as angiotensin converting enzyme inhibitors and angiotensin II receptor blockers may be helpful in the treatment of patients by improving endothelial dysfunction [21].

\section{Limitations of the study}

Our study had several limitations that warrant consideration. The primary limitation was that our study was a nonrandomized and single center study with a limited number of patients. A study involving more patients could have more significant results. In addition, anatomical imaging modalities, including intravascular ultrasound or optical coherence tomography to assess the ectatic vessels were not used.

\section{Conclusion}

Increased ADMA levels may play an important role in the pathogenesis of diffuse CAE. Further large-scale studies are required to determine the mechanism of this relationship between ADMA levels and CAE. These findings suggest that increased ADMA level may be associated with endothelial dysfunction leading to the development of CAE and therapeutic strategies targeting endothelial dysfunction may be useful in the management of patients with CAE.

\section{Conflict of interest}

All the authors have no conflict of interest.

\section{References}

1. Swaye PS, Fisher LD, Litwin P, Vignola PA, Judkins MP, et al. (1983) Aneurysmal coronary artery disease. Circulation 67: 134-138.

2. Pinar Bermúdez E, López Palop R, Lozano Martínez-Luengas I, Cortés Sánchez R, Carrillo Sáez P, et al. (2003) Coronary ectasia: prevalence, and clinical and angiographic characteristics. Rev Esp Cardiol 56: 473-479. [Crossref]

3. Sarli B, Baktir AO, Saglam H, Arinc H, Kurtul S, et al. (2014) Neutrophil-tolymphocyte ratio is associated with severity of coronary artery ectasia. Angiology 65 : 147-151. [Crossref]

4. Mavrogeni S (2010) Coronary artery ectasia: from diagnosis to treatment. Hellenic $J$ Cardiol 51: 158-163. [Crossref]

5. Aktürk E, Askin L, Nacar H, Taşolar MH, Türkmen S, et al. (2018) Association of serum prolidase activity in patients with isolated coronary artery ectasia. Anatol J Cardiol 19: 110-116. [Crossref]
6. Stajduhar KC, Laird JR, Rogan KM, Wortham DC (1993) Coronary arterial ectasia: increased prevalence in patients with abdominal aortic aneurysm as compared to occlusive atherosclerotic peripheral vascular disease. Am Heart J 125: 86-92. [Crossref]

7. Vallance P, Leone A, Calver A, Collier J, Moncada S (1992) Endogenous dimethylarginine as an inhibitor of nitric oxide synthesis. J Cardiovasc Pharmacol 20: 60-62. [Crossref]

8. Landim MBP, Casella Filho A, Chagas ACP (2009) Asymmetric dimethylarginine (ADMA) and endothelial dysfunction: implications for atherogenesis. Clinics 64: 471478. [Crossref]

9. Böger RH (2003) The emerging role of asymmetric dimethylarginine as a novel cardiovascular risk factor. Cardiovasc Res 59: 824-833. [Crossref]

10. Markis JE, Joffe CD, Cohn PF, Feen DJ, Herman MV, et al. (1976) Clinical significance of coronary arterial ectasia. Am J Cardiol 37: 217-222. [Crossref]

11. Landmesser U, Drexler H (2005) The clinical significance of endothelial dysfunction. Curr Opin Cardiol 20: 547-551. [Crossref]

12. Chrissoheris MP, Donohue TJ, Young RS, Ghantous A (2008) Coronary artery aneurysms. Cardiol Rev 16: 116-123. [Crossref]

13. Neunteufl T, Katzenschlager R, Hassan A, Klaar U, Schwarzacher S, et al. (1997) Systemic endothelial dysfunction isrelated to the extent and severity of coronary artery disease. Atherosclerosis 129: 111-118. [Crossref]

14. Davignon J, Ganz P (2004) Role of endothelial dysfunction in atherosclerosis. Circulation 109: III27-32. [Crossref]

15. Vallance P (2001) Importance of asymmetrical dimethylarginine in cardiovascular risk. Lancet 358: 2096-2097. [Crossref]

16. Mavrogeni S (2010) Coronary artery ectasia: from diagnosis to treatment. Hellenic $J$ Cardiol 51: 158-163. [Crossref]

17. Ramappa P, Kottam A, Kuivanemi H, Thatai D (2007) Coronary artery ectasia--is it time for a reappraisal? Clin Cardiol 30: 214-217. [Crossref]

18. Yilmaz H, Sayar N, Yilmaz M, Tangürek B, Cakmak N, et al. (2008) Coronary artery ectasia: clinical and angiographical evaluation. Turk Kardiyol Dern Ars 36: 530-535. [Crossref]

19. Morrad B, Yazici HU, Aydar Y, Ovali C, Nadir A (2016) Role of gender in types and frequency of coronary artery aneurysm and ectasia. Medicine (Baltimore) 95: e4395. [Crossref]

20. Malviya A, Jha PK, Mishra A (2017) Isolated coronary artery ectasia: Clinical angiographic, and follow up characteristics. Indian Heart J 69: 619-623. [Crossref]

21. Delles C, Schneider MP, John S, Gekle M, Schmieder RE (2002) Angiotensin converting enzyme inhibition and angiotensin II AT1-receptor blockade reduce the levels of asymmetrical $\mathrm{N}(\mathrm{G}), \mathrm{N}(\mathrm{G})$-dimethylarginine in human essential hypertension. Am J Hypertens 15: 590-593. [Crossref]

Copyright: (C2019 Çelik O. This is an open-access article distributed under the terms of the Creative Commons Attribution License, which permits unrestricted use, distribution, and reproduction in any medium, provided the original author and source are credited. 